https://artnodes.uoc.edu

\title{
Imaginando ecologías futuras: la imaginación kantiana a través de las generaciones ${ }^{1}$
}

\author{
Irene Gómez Franco \\ Universidad Autónoma de Barcelona
}

Fecha de presentación: octubre de 2021

Fecha de aceptación: enero de 2022

Fecha de publicación: enero de 2022

\section{Cita recomendada}

Gómez Franco, Irene. 2022. «Imaginando ecologías futuras: la imaginación kantiana a través de las generaciones». En: Garcés, Marina (coord.). «Ecología de la imaginación». Artnodes, no. 29. UOC. [Fecha de consulta: dd/mm/aa]. https://doi.org/10.7238/artnodes.v0i29.393022

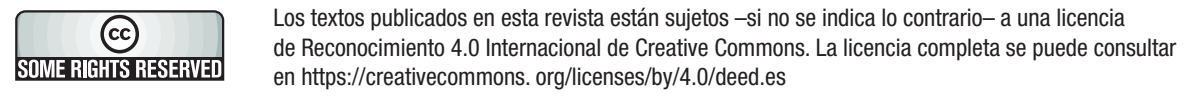

\begin{abstract}
Resumen
La imaginación es una facultad que permite al ser humano ponerse en el lugar del otro y tener presentes diferentes puntos de vista. En este artículo examino de qué manera la imaginación puede concebirse en una dimensión ampliada no solo en el espacio sino también en el tiempo, en el sentido de una «visita al otro» futuro. Para este fin, primero examino diversos ejercicios de la imaginación: en tanto que simpatía tal y como la formuló Adam Smith y compromiso según Amartya Sen, pero también en la lectura política que Hannah Arendt realiza de la facultad de juzgar de la filosofía kantiana. Esta idea de la imaginación es promisoria para fundar una responsabilidad basada en lo público y en la deliberación orientada a la construcción del mundo en común, haciéndonos cargo de las necesidades de las generaciones futuras. Pero la imaginación por sí sola no es suficiente, se
\end{abstract}

1. Este artículo ha sido realizado en el marco de los proyectos internacionales: «Tendiendo puentes: justicia intergeneracional y cosmopolita en tiempos de pandemia (WORLDBRIDGES II)» e «NNconRES: Incertidumbre, confianza y responsabilidad. Claves ético-epistemológicas de las nuevas dinámicas sociales (en la era digital)», PID2020-117219GB-I00), Ministerio de Ciencia e Innovación en el marco del Plan Estatal l+D+i del Instituto de Filosofía del Consejo Superior de Investigaciones Científicas. La autora agradece a Astrid Wagner y María Teresa Muñoz Sánchez sus valiosos comentarios, a dos revisores externos por sus acertadas sugerencias y a todos/as los miembros de MUSSOL por sus lúcidas observaciones a una versión borrador de este artículo. 


\title{
artnodes
}

https://artnodes.uoc.edu

Imaginando ecologías futuras: la imaginación kantiana a través de las generaciones

necesita también una educación del individuo, a la vez que estructuras políticas y económicas que fomenten una

ciudadanía activa, crítica y responsable.

\section{Palabras clave}

imaginación; juicio político; Immanuel Kant; Hannah Arendt; futuras generaciones

Imagining future ecologies: Kantian imagination across generations

\begin{abstract}
Imagination is a faculty which enables human beings to put themselves in the place of another and bear in mind differing points of view. In this article, I examine the ways in which imagination can be conceived of in an expanded dimension, not just in space but also in time, in the sense of a future "visit to the other". To this end, I will first examine various imaginative exercises: in terms of kindness as formulated by Adam Smith and of commitment according to Amartya Sen, but also in the appropriation that Hannah Arendt performs of the faculty of judgement in her political lecture on Kantian philosophy. This idea of the imagination is promising for creating a responsibility based on what is public and on deliberation orientated towards the construction of a common world, assuming responsibility for the needs of future generations. But imagination alone is not enough; individual education is also needed along with political and economic structures that foster active, critical, and responsible citizenship.
\end{abstract}

\section{Keywords}

imagination; political judgement; Immanuel Kant; Hannah Arendt; future generations 


\section{artnodes}

https://artnodes.uoc.edu

\section{Introducción}

A los integrantes de la Convención Ciudadana por el Clima (CCC) que tuvo lugar en París entre los años 2019 y 2020 se les pidió que debatieran y consensuaran una serie de medidas vinculantes para el Gobierno dirigidas a reducir en diez años y en un $40 \%$ los niveles de emisión de gases de efecto invernadero en el territorio nacional. La Convención estaba compuesta por 150 ciudadanos y ciudadanas escogidos mediante un sorteo después de haber definido una serie de criterios como la edad, el territorio y el nivel de estudios, con el objetivo de obtener una muestra representativa de la sociedad francesa. ${ }^{1}$

Con vistas a fomentar el debate informado y la identificación de propuestas factibles y eficaces, uno de los elementos clave de dicho experimento fue la formación que recibieron los integrantes en el estadio inicial. Estos se reunieron con expertos que argüían opiniones contradictorias, estudiaron informes de organismos internacionales, análisis de expertos en industria, agricultura y transporte, y analizaron resúmenes de trabajo elaborados por colectivos de la sociedad civil. No se trataba, en esencia, de un encuentro de especialistas, sino de reunir las necesidades de personas que no cuentan con conocimientos específicos en materia de cambio climático. Acompañadas de profesionales del derecho y de expertos en diálogo ciudadano, fueron capaces de acordar un proyecto de ley; 149 propuestas con un estudio de financiación y de reforma de la Constitución que iban, en un principio, a ser objeto de un referéndum o una votación en el Parlamento sin ser filtradas, según lo prometido por Emmanuel Macron.

Muchos elementos de peso, potencialmente atractivos para el análisis, coadyuvaron al éxito de la Convención a pesar de que algunas lecturas hayan interpretado la iniciativa como experimento fallido dada la lenta reacción del presidente de la República en el plano legislativo y la adopción de únicamente un $40 \%$ de las propuestas de la Convención. Se ha llegado a pensar que se trataba más bien de una estrategia dirigida a apaciguar a la opinión pública tras el descontento y las revueltas de los chalecos amarillos. No obstante, desde mi óptica, el experimento de democracia participativa ha sido uno de los más reveladores de los últimos años en nuestro continente, en tanto que pone sobre la mesa la fecundidad de educar el juicio crítico, la llamada inteligencia colectiva y, en particular, la imaginación, algo que se traslada a acciones políticas situadas y plurales en cuanto que abrazan la diversidad humana y la diferencia de puntos de vista. Puntos de vista que sin despojarse de sus raíces 0 circunstancias personales son capaces de pensar ecologías futuras no obstante la incertidumbre. Muestra, por tanto, que se puede llegar a acuerdos y que la imaginación, como facultad que va de la mano del juicio y de la deliberación crítica, puede ser llave de paso para una reconstrucción y cuidado de lo común.
Imaginando ecologías futuras: la imaginación kantiana a través de las generaciones

De eso se trataba cuando se invitó a los integrantes de la Convención a unirse al panel. Se les interpelaba a pensar desde sus lugares, pero, a la vez, en las fronteras. Las crisis que experimentamos nos sitúan en un espacio que nos permite vérnoslas con las fronteras, y en estas fronteras, como límites, podemos habitar y pensar paisajes alternativos y radicales. Los límites son limitantes, nos sitúan en los confines del saber y experimentar, alertándonos de que actividades y dinámicas sistémicas no pueden seguir desenvolviéndose como habían venido haciéndolo hasta ahora. Pero también son posibilitantes. Lo que tenían que hacer era imaginar y, a partir de esa actividad, concebir ecologías nuevas, considerando un «tro» en lo individual y en lo colectivo. Las fronteras y los límites dan alas a la imaginación 0 , tal y como lo formula Marina Garcés, «la imaginación es la facultad de los límites: una actividad sensible y mental que nos relaciona de forma activa con los límites de lo que vemos, sabemos o pensamos». Esta imaginación es una facultad crítica que reúne «lo sensible y lo inteligible, lo interior y lo exterior, lo presente y lo ausente, lo que es y lo que no es, lo posible y lo imposible... y las distintas dimensiones del tiempo» (Garcés, 2022. En este número).

\section{Juzgar políticamente, imaginar colectivamente}

En las líneas anteriores, he establecido, aunque sin entrar en detalle, una relación entre el juicio y la imaginación. A partir de esta ligazón he arrojado la posibilidad de una proyección hacia algo que he denominado ecologías futuras. ${ }^{2}$ Entiendo las ecologías futuras como mundos y ecosistemas relacionales que, por su adjetivo, están por venir.

Centrándonos ahora en los juicios, de entre los tipos de juicio existentes, los políticos son de una clase que adquiere validez y efectividad en el momento en que se hace presente a los demás. Y, como veremos a continuación, el medio que hace esto viable es la imaginación. De aquí el interés particular en este enlazamiento productivo entre un tipo de juicio y la facultad de imaginar, que es la base para la facultad de juzgar. Para ello, hay que entender el juicio en su sentido amplio y, en estas, como la facultad que organiza y otorga coherencia a la experiencia humana -a lo que Immanuel Kant llamaba «modo de pensar amplio» (erweiterte Denkungsart)-, sea en su vertiente lógico-cognitiva cuando «se trata de particulares de objetos que es necesario relacionar con conceptos", sea para los objetivos de la vida política, cuando es necesario ordenar "particulares de acontecimientos [...] en narrativas" (Zerilli 2008, 242-243). Desde el momento en el que vemos un «pájaro» en el balcón, o leemos una noticia en el periódico matutino acerca de "la llegada de más inmigrantes en pateras a las costas españolas», hasta el discurso de un representante de un partido político que

\footnotetext{
1. Merece la pena visitar la página de la CCC para conocer los detalles del proceso y sus resultados: https://www.conventioncitoyennepourleclimat.fr/
}

2. No efectúo aquí distinción entre la facultad de juzgar o discernir (según la traducción que se use) y el producto de esta, el juicio. 


\section{artnodes}

https://artnodes.uoc.edu

Imaginando ecologías futuras: la imaginación kantiana a través de las generaciones

escuchamos en la radio sobre «la regulación del mercado eléctrico», somos receptores y protagonistas de la práctica de juicio que impregna todas las actividades humanas. El juicio es sustantivo para el ser humano porque es lo que le permite que el mundo adquiera un sentido. Incluso la mera cognición no es reducible a sensación o sensibilidad (Empfindung). El juicio es la capacidad de pensar que lo particular está contenido en lo universal: «consiste en la capacidad de subsumir bajo reglas, es decir, de distinguir si algo cae o no bajo una regla dada» (Kant 1996, 179, B171). Sin esta operación en la que la experiencia cae bajo reglas (conceptos que clasifican los particulares) no podríamos tener conocimiento, ni nada que se pudiera considerar un objeto, ni siquiera un «esto». Se torna evidente, solo considerando los conceptos más generales, que, desde la conceptualización kantiana, sin las categorías no sería posible siquiera una individualización de un objeto como «esto»; en su lugar, tendríamos una corriente de sensaciones no estructuradas.

Para la idea de juicio es basilar la distinción que Kant establece entre juicios determinantes y juicios reflexionantes, distinción altamente productiva y que dará lugar a una lectura política de la filosofía kantiana por parte de Hannah Arendt. ¿Cuál es la diferencia entre ambos tipos de juicios? Pues bien, del primer tipo serían los juicios cognoscitivos o lógicos, donde la regla, la ley o el principio está dado y solo cabe subsumir lo particular a lo universal. No obstante, en contraste con estos, Kant utiliza otro tipo de juicio en la tercera crítica, en la Crítica del juicio, el juicio reflexionante, donde lo dado es lo particular y la capacidad de juicio tiene que buscar el universal.

Aquí es donde se sitúa Arendt. Las reivindicaciones y demandas de carácter político serán ejemplos de estos juicios reflexionantes, como lo son los juicios estéticos o del «gusto». A modo de ilustración, cuando afirmamos que la vida de los animales no humanos debe ser respetada y no convertida a mera cosa, lo que estamos afirmando no es una cuestión epistemológica de un conocimiento de los particulares; antes bien, nos estamos situando en el ámbito de lo nuevo que aparece en tanto que cuestión política. ${ }^{3}$ Pero, esta forma distinta de pensar tiene una exigencia peculiar, nos pide que salgamos de nuestra autoconsciencia para pensar poniéndonos en el lugar de los demás, para, tomando prestada la expresión de Arendt, «ir de visita» a los otros, lo que es posible gracias a la imaginación [Einbildungskraff]. Es decir, juzgar políticamente y llegar a un acuerdo potencial, «compartir-el-mundocon-los-demás", requiere imaginación. Efectivamente, se necesita una facultad que pueda mediar entre lo abstracto y lo particular, y entre lo individual y colectivo, que pueda comunicarse y compartirse, una facultad que también sea radical y transformadora.

No olvidemos que la teórica alemana se vio interpelada a revisar la categoría de juicio en el momento en el que los criterios y paradigmas heredados habían desembocado en el evento político definitivo del siglo
XX: el totalitarismo. Por ello, una cuestión que cautiva el pensamiento de Arendt es el problema de la validez y la verdad; de cómo, para que el pensamiento político sea representativo y válido, se precise considerar un tema desde diferentes ángulos haciendo presentes, hasta tal punto, los criterios de los ausentes, representándolos, en un juego en el que el individuo no se disfraza de otra identidad, ni abandona la suya propia, ni se une a las filas de manera ciega de otros puntos de vista. Tampoco es empatía, dirá Arendt, sino, más bien, ser y pensar aún con mi identidad, «Como en realidad no soy» $(2016,369)$.

Cuantos más puntos de vista diversos tenga yo presentes cuando estoy valorando determinado asunto, y cuanto mejor pueda imaginarme cómo sentiría y pensaría si estuviera en lugar de otros, tanto más fuerte será mi capacidad de pensamiento representativo y más válidas mis conclusiones, mi opinión (Idem).

Es la presencia de las perspectivas de los demás lo que graba la validez del juicio. Necesitamos los ejemplos para urdir reglas que nos guíen en el reconocimiento de lo correcto y lo incorrecto ante lo que no tenemos a priori un concepto. Cuantos más puntos de vista pueda representarme para juzgar un problema, más sólida será mi capacidad de juzgar y más válidas serán mis conclusiones; más fuerte mi capacidad de ponerme en el lugar del otro. En último término, la validez se origina de este mundo compartido que es la humanidad, la comunidad del mundo, hasta el punto de que los sistemas democráticos peligran, no por sistemas de pensamiento de lo absoluto, sino por la pérdida de ese mundo en común (Muñoz Sánchez 2017, 527).

En este mundo, nuestros juicios entrarán en juego con el resto de los juicios para encontrar un consenso cortejado donde la búsqueda de la verdad puede ser, cuando menos, contraproducente. La política no es acerca de la verdad, dice Arendt. El debate es lo intrínseco a la política, el hacer las cosas públicas. Por ello, hay que dejar de lado los sueños de la universalidad para, a través de la persuasión y el convencimiento, discriminar opiniones, comunicar y acordar.

El «ponerse en el lugar de los otros» se perfila como substancial en la vida en común del ser humano. Este siente un profundo desasosiego en cuanto que ansía formar parte de lo común y reconocerse en los demás, porque la comunidad del mundo es el lugar de los lazos y no de los seres solitarios, y es el lugar de la pluralidad de los juicios y de la interrelación dialógica con los otros.

\section{De visita al otro}

En La teoría de los sentimientos morales, Adam Smith se muestra preocupado por la popular interpretación de los afectos humanos que de manera incorrecta asevera que todas las acciones humanas se nutren

3. En este caso, los derechos de los animales no humanos son un tema que genera en el ámbito jurídico, político y ético mucha controversia, ya que no contamos todavía con principios y leyes acordados social y públicamente de manera definitiva y exhaustiva. Vamos dando pasos hacia una ampliación de esos derechos -amparados por la ley- y de nuestra concepción de sus necesidades y sus capacidades, su dignidad y su florecimiento, pero todavía falta mucha discusión, teoría, leyes y prácticas de respeto y reconocimiento. 


\section{artnodes}

https://artnodes.uoc.edu

del egoísmo. Le preocupa que se conciba a la humanidad como capaz únicamente de buscar el interés propio, siendo imposible superarlo sin obtener un beneficio personal cuando, señala, el «amor propio» no es el único componente del comportamiento del individuo, sino que también son sustantivos sentimientos como la simpatía, la generosidad y el espíritu público. Ante este vicio, la facultad de la imaginación nos permite efectuar un «cambio de papeles» como lo describirá Smith. Al cambiar los papeles no lo hago yo en la medida de mi persona y mis características, sino en tanto «yo» como «tú mismo». Es decir, me imagino cómo me encontraría yo ante una tragedia, un infortunio o una alegría, si yo fuera tú, teniendo lugar así este viaje, no en mí, sino en tu persona.

Cuando me duelo por la muerte de su único hijo, con objeto de identificarme con su aflicción, no pienso en lo que yo mismo, una persona con tales características y profesión sufriría si tuviese un hijo y si ese hijo desgraciadamente muriese; lo que hago es considerar cuánto sufriría yo si yo fuese en realidad usted, y no sólo cambio con usted el contexto sino también las personas y los caracteres. Mi pesadumbre entonces, obedece por entero a su causa y en nada a la mía. Por consiguiente, no es egoísta en absoluto. ¿Cómo puede calificarse de egoísta una pasión que ni siquiera en la imaginación brota de nada que me haya sucedido o que tenga que ver conmigo, con mi persona y carácter, sino que sólo le atañe lo que tiene que ver con usted? (Smith $2009,538){ }^{4}$

De nuevo se pone de relieve el papel basilar que tiene la imaginación como facultad facilitadora de este «cambio de papeles» 0 de esa «visita al otro». La imaginación es fundadora de esa posibilidad de tener presentes los padecimientos, como apuntaba Smith, o las lecturas plurales a un problema desde el punto de vista interpretativo, como afirmaba Arendt. Tener presente lo que, incluso en las manifestaciones del ser, se encuentra ausente conlleva la capacidad de transportarnos a lugares y personas que no tienen que encontrarse aquí y en el instante, ni ser parte de la comunidad a la que pertenecemos. ${ }^{5}$

Lo que nos proporciona esta visita es una objetividad que de otra manera sería difícil de conseguir. El riesgo de la cortedad de miras, deseos y juicios moldeados acríticamente en una comunidad cerrada no es buen consejero a la hora de construir lo público. Los juicios de una determinada comunidad no tienen por qué ser correctos tal y como ya puso de relieve Arendt en su momento. La objetividad que nos interesa no es una cualidad lógica, como sería el principio de no contradicción, sino esa habilidad de juzgar con la mirada de todos los demás emplazando la atención, antes que en la razón 0 en el entendimiento, en la imaginación. Es esta condición de objetividad, entonces, la que se está buscando para que el pensamiento crítico y nuestra producción de mundo hagan posible una comunidad mundial o una existencia cosmopolita, tomando prestadas las expresiones arendtianas.
Imaginando ecologías futuras: la imaginación kantiana a través de las generaciones

Ahondando más en la cuestión, cabe sacar a colación que la simpatía en la concepción de los sentimientos morales del ilustrado escocés, que permiten esta identificación y transmisión de afectos, también favorece ser conscientes de ellos en el momento en que la razón se pregunta las causas, dado que se incluye la situación o el motivo en el que dicha pasión se originó. En otros términos, abre un espacio a juzgar mis propios actos y motivaciones a la vez que juzgo las acciones e inclinaciones de los demás. La simpatía es crítica y, a la vez, autocrítica.

Solo es posible que se pueda hablar de un comportamiento correcto 0 incorrecto si salimos de nosotros mismos y nos vemos según lo harían los otros. Por eso, Arendt le da tanta importancia a la figura del espectador que parecería coincidir con la idea brillantemente esculpida del espectador imparcial de Adam Smith. El espectador imparcial es fruto del juego de la imaginación en el que se necesitan actores que a la vez serán espectadores de nuestro actuar. 0 , en otras palabras, significa incluir la perspectiva social de la persona que observa, porque la simpatía no se reduce a un proceso mental dentro de los límites de la razón individual como la concibió David Hume (Wences Simón 2007, 29-30).

Esta idea viene a ser recuperada magistralmente por Amartya Sen bajo su noción del compromiso. En comportamientos habituales, explica Sen, podemos apreciar que ciertas acciones que realizan los individuos parecen ir incluso en contra de su interés o bienestar. Esto significa ir más allá de la simpatía porque esta, al fin y al cabo, no está reñida con el «amor propio»; mi bienestar incluye el tuyo. De acuerdo con Sen, cuando nos movemos impulsados por el compromiso lo hacemos porque juzgamos que una acción es justa 0 es correcta y esta acción no va a reportarme necesariamente un bienestar mayor que el que tenía antes de la acción. Por bienestar se entiende, desde el punto de vista de las teorías económicas clásicas del interés y las preferencias que son el foco de atención del pensador bengalí, que vamos a ir en contra de nuestra maximización de las preferencias, lo que es idéntico a afirmar que no estamos maximizando nuestro interés.

Por tanto, el compromiso es lo que explica que llevemos a cabo acciones que no tienen una repercusión necesariamente positiva en términos de bienestar para nosotros. Algunos comportamientos que tienen como fin construir una vida más ecológica y sostenible son de este tipo: reducir los viajes en avión para trayectos que son accesibles en tren 0 autobús sería un ejemplo típico. Considérese que, en estos casos, estamos actuando de un cierto modo porque lo consideramos correcto o justo renunciando a otra opción del actuar que nos reportaría, según el utilitarismo de las preferencias, un bienestar mayor. Los fines altruistas 0 de justicia pasan a ser engullidos por la tesis rígida del egoísmo en tanto que, en definitiva, eso es lo que preferimos: no vamos a ir nunca en contra de lo que maximiza nuestro bienestar, nuestra utilidad. Sin embargo, ayudar a una persona porque estoy convencida de que es lo justo, siendo

4. Cursiva añadida por la autora del artículo.

5. Idea esta que desarrolla magistralmente Seyla Benhabib en su obra Los derechos de los otros con respecto a un universalismo cosmopolita transfronterizo (2015). 


\section{artnodes}

https://artnodes.uoc.edu

Imaginando ecologías futuras: la imaginación kantiana a través de las generaciones

este el motivo de mi actuar, no implica que el aumento de bienestar de esa persona proporcione un incremento directo en mi bienestar, puede incluso reportarme un nivel de bienestar más bajo que el que me pudiera producir otra alternativa (Sen 1977, 327).

Entiendo que ciertas acciones explicadas desde el compromiso no solo tienen cabida, sino que son fundamentales en nuestro habitar en el mundo con los otros. Es más, el compromiso es promisorio en cuanto a través de la imaginación me comprometo con la humanidad no presente temporalmente, con la humanidad futura.

De lo visto hasta ahora, si se quiere pergeñar una disección analítica, nos encontraríamos con tres niveles de ejercicio de la imaginación en su transitar del sujeto a los otros. Uno sería el comportamiento egoísta 0 basado en el amor propio, otro sería aquel impulsado por la simpatía y un tercero nos conduciría a los dominios del compromiso. Pero de estos tres el imaginar como compromiso es lo que nos orienta hacia un pensamiento que cruza la nación, la comunidad, la cultura y el tiempo. Aún más, me interesa el carácter político y no solo moral de este «ponerse en el lugar del otro», y por ello, la idea arendtiana de la imaginación y el juicio cobra especial relevancia. Hay que «ver políticamente», sentenciará Arendt (1993b, 96 Z), en tanto ejercicio imaginativo que nos impulsa a crear conceptos políticos ahí donde las viejas categorías no tienen ya fuerza explicativa.

\section{Imaginación ampliada y futuras generaciones}

Por muy fecunda que resulte la apropiación de la idea de imaginación y juicio de Hannah Arendt en su lectura política de Kant, algo que no encontramos en su estudio y que lamenta Linda Zerilli, es el tránsito de la imaginación como re-presentación a poder de producción $(2011,127)$. Para nuestros fines, se precisa una facultad que sea radical y transformativa, a la vez que compartida. Por ello nos interesa especialmente otra expresión de la imaginación que sea capaz de crear relaciones entre cosas aparentemente distantes, esto es, la imaginación productiva en contraste con una imaginación meramente representativa. ${ }^{6}$

¿Cómo crear formas, conceptos y relaciones que no se sometan a lo existente? Si lo nuevo tuviera que subsumirse bajo la categoría de lo conocido o experimentado, entonces significaría que a lo novedoso se le encaja en lo viejo como requisito para poder ser pensado y comprendido. Pero la imaginación es también generadora, no está atada a las leyes de causalidad o temporalidad, puede juzgar sin concepto, sin caer bajo las leyes del entendimiento, sin ser forzada a formular encadenadamente juicios cognitivos. La expresión de la imaginación en cuanto que productiva actúa en libertad, como Kant ya mostró que sucede en los juicios estéticos. Pero en esta dirección no es solo capaz de crear y de hacer presente algo nuevo, como el considerado genio artístico, sino que también lo es la imaginación como facultad política. Es la imaginación generativa la que produce conceptos políticos que modifican nuestra idea de mundo y descubren lo que nos es común y lo que nos separa.

Toda extensión de un concepto político implica siempre una apertura imaginaria del mundo que nos permite ver y articular relaciones entre cosas que no tienen relación alguna (en ningún sentido lógico, necesario), crear relaciones que son externas a sus términos. Las relaciones políticas siempre son externas a sus términos: no implican tanto la capacidad de subsumir particulares bajo conceptos sino más bien un elemento imaginativo: la capacidad de ver o forjar nuevas conexiones (Zerilli 2008, 308).

Esta habilidad de crear nuevas conexiones nos da acceso a entender la imaginación en su forma ampliada: imaginando para ver también las prioridades y necesidades de otras personas, extendiéndola, incluso, a través de generaciones. Así se entiende cuando se habla en el campo ético-político de justicia intergeneracional, sin ir más lejos. Porque la justicia intergeneracional es un tipo de justicia global, podemos aplicar la imaginación y el discernimiento crítico no solo para considerar una igualdad cosmopolita y global, sino también para pensar la justicia intergeneracional en su traslación concreta a obligaciones que se dilatan hasta abarcar la calidad de vida de las futuras generaciones.

Si nos tomamos en serio el impacto y las dimensiones de las consecuencias de la acción humana, las categorías tradicionales con las que comprendemos y nos representamos el mundo se revelan insuficientes como sucede con la categoría de la responsabilidad. № obstante, estas ecologías futuras nos demandan una responsabilidad que no solo tiene en cuenta nuestro hoy, sino que nos hace responsables del mañana. ${ }^{7}$ Responsabilidad, del latín respondere, de spondere (prometer) y re- (reiteración). Este responder conduce a la «semántica ética (y, por lo tanto, constitutivamente relacional) de la promesa entendida como peño» (Marramao 2012, 34). Em-peñarse con alguien 0 algo adquiere una dirección en cuanto es con el otro, y por ello es en esta dimensión interrelacional que parte de nuestra libertad. Imaginar nos responsabiliza con el otro, con el mundo, aunque se torna evidente que en la responsabilización el otro no tiene por qué estar presente. Es esta nueva acepción de la responsabilidad la que andamos buscando, la cual viene ayudada por la imaginación con sus funciones representativa y generadora.

Ahora bien, aun reconociendo que podemos tener un deber hacia las generaciones futuras, resulta complejo construir un mapa de deberes y acciones que transformen el presente considerando el tipo de vida que las personas llevarán en el futuro. Es un problema de larga

6. Linda Zerilli afirma que esta idea se encuentra ya en la edición A de la Crítica de la razón pura de Kant y que se puede ver en la idea kantiana de «ejemplo», el cual actúa de la misma manera que el «esquema» $(2011,127)$.

7. Me he dedicado a la cuestión de la responsabilidad intergeneracional y los deberes que tenemos hacia las generaciones futuras en el volumen Deudas pendientes. La justicia entre generaciones (Gómez 2020). Invito al lector o lectora interesada en profundizar estas ideas a consultar dicha obra. 
data que nos hallamos mayormente implicados con los que están más cerca que con los que no forman parte de nuestro círculo sentimental, nuestro país, nuestra cultura; un problema con el que ya se las ha visto y se las sigue viendo la idea de la justicia internacional o transfronteriza. Pero las decisiones que se toman en la actualidad a nivel político, social y económico tienen efectos a largo plazo que inciden y, en ocasiones, erosionan las condiciones de vida de personas del futuro, sin contar nuestra convivencia con otras formas de vida que están siendo extinguidas 0 ven sus vidas reducidas e instrumentalizadas. Resulta evidente, entonces, que nuestra responsabilidad no puede limitarse a las generaciones convivientes, ni siquiera a los derechos y calidad de vida de nuestros hijos y nietos, sino que debe adoptarse una actitud que supere el cortoplacismo (o la idiotez emocional) para proyectarse hacia el futuro. Las generaciones futuras no pueden decidir, no tienen ni voz ni voto, aunque nosotros estemos determinando sus opciones de vida.

El espíritu cortoplacista de algunas propuestas de los Gobiernos es un síntoma de esta falta de compromiso y responsabilidad. El cortoplacismo nos es bien conocido; vivimos atados al cortoplacismo. El consumo de imágenes, contenidos e información es instantáneo. Los productos tecnológicos se vuelven obsoletos en tiempos brevísimos. Las operaciones financieras se cuentan en nanosegundos. El cortoplacismo es un peligro cuando se trata de problemas urgentes de calado social y vital con una vigencia a largo plazo como es el cambio climático. Ante esto, también somos capaces de pensar proyectivamente, originar planes de sostenibilidad y espacios de libertad. Por tanto, este pensamiento con horizontes dilatados no nos es completamente ajeno, como sostiene el filósofo Roman Krznaric con su idea del pensamiento catedral, pues el ser humano es autor de grandes obras construidas a lo largo de muchísimos años.

Lo que queda por ver es si la imaginación por sí sola es suficiente. ¿Qué herramientas y estrategias nos ayudan a cultivar esa imaginación y una perspectiva ampliada sobre los deseos y prioridades de las generaciones futuras de tal manera que podamos tomar decisiones correctas?

Martha Nussbaum (2010) ya señaló que la imaginación es un don, pero que necesita pulirse, educarse. Las artes y las humanidades tienen un papel central en esta educación con vistas a sociedades democráticas reflexivas, dialogantes, plurales y saludables.

Lo que Nussbaum denomina «imaginación narrativa» resulta capital para la construcción de una ciudadanía mundial; una formación de ciudadanos que sea capaz de ver el mundo a través de los ojos de otras personas. Esta capacidad requiere que el ser humano se dé cuenta de su vulnerabilidad, pero también que tenga en cuenta la de los otros percibiendo que no está solo en el mundo y que existimos en codependencia. Las artes y las humanidades son esenciales a la hora de formar la comprensión que el individuo tiene de sí mismo, y de las expectativas y fragilidades de los demás. El cultivo de los recursos imaginativos unidos a los emocionales fomenta la sensibilidad y el cuidado de nuestro propio mundo interior, así la comprensión y la comunicación, a la vez que permite arrojar luz en los puntos ciegos y dogmáticos de una cultura 0 una tradición.

Ahora bien, el cultivo de la imaginación necesita otros ingredientes. ¿Cómo va a pensarse en lo común presente y futuro sin la práctica política con el otro? Es decir, este sujeto que imagina tiene que ser capaz de deliberar e imaginar colectivamente, pues existe una retroalimentación del juicio que solo se produce en la acción con el otro. Ahora que la idea del cuidado goza de tan buena salud -un cuidado que entiendo como ética democrática y humana, no un cuidado bajo la lógica del sistema de explotación neoliberal y patriarcal-, cuidar de lo otro significa también usar esta imaginación. Es un cuidar activo, un cuidar como práctica política. Compartir mundo se puede imaginar y, así, se puede dar. Como bien afirma Camps:

«Hacer del cuidado un objetivo político [...] significa diseñar estructuras que propicien la redistribución de las obligaciones de cuidarnos mutuamente. Significa asimismo tomarse en serio la llamada "transición ecológica" y hacer del cuidado de la "casa común" una preocupación sostenida y prioritaria» (2021).

Nótese que la filósofa, con esta idea de cuidado, está apuntando a la necesidad de contar con estructuras que promuevan ese cuidado como ejercicio democrático. No parece que sea suficiente la acción de comunidades e individuos volcados en cuidar lo común, siempre que no haya un compromiso político firme de Gobiernos e instituciones que controlan las opiniones y las libertades de las personas. Y es que la imaginación acompañada de la pasión y el compromiso, y de la acción del juicio deliberativo, tiene que encontrar eco y trasvase en el modo de hacer y en las medidas que toman las esferas que controlan y ostentan el poder económico e institucional. Claramente, falta en lo relativo a cuestiones políticas de primer orden, como es el cambio climático, una planificación eficaz y real con fronteras y horizontes amplios e intergeneracionales.

\section{Conclusiones}

Algo que ya han señalado los teóricos del enfoque de las capacidades es que, para contar con sociedades justas 0 corregir debidamente las injusticias que viven las personas, hay que poner el acento en el papel que tienen los individuos 0 en el que tienen las instituciones. Para Nussbaum, la relación entre una imaginación compasiva y las instituciones sociales es bidireccional: los ciudadanos y las ciudadanas construyen sistemas institucionales que son reflejo de lo que imaginan; a su vez, las instituciones influyen en el desarrollo de la compasión de las personas, en el fomento de actitudes cívicas, de mutuo reconocimiento y de responsabilidad. Las instituciones, además, pueden alentar o combatir ciertas emociones que pueden llegar a ser perjudiciales para el desarrollo de la imaginación en su vertiente de juzgar y formar seres humanos responsables, como serían la vergüenza, la envidia y el asco (Nussbaum 2008, 449). Para terminar, téngase en cuenta que incluso 
la posición desde donde se imagina, cuenta, y que en este desempeño habría que considerar cómo la memoria intercede y se implica conduciendo nuestras representaciones y juicios en una dirección u otra.

De muchas maneras y en muchos niveles se puede, institucionalmente, dirigir estrategias que antes de priorizar los intereses personales se impliquen en promover ese ejercicio de la imaginación en su vertiente colectiva con un compromiso fuerte de traspasar los resultados y las propuestas al ámbito jurídico. Algunas de ellas sin duda ya están teniendo lugar -hemos abierto esta exposición con una que nos resultaba bastante ilustrativa, la CCC-. La ciudadanía sigue pidiendo mayor implicación, apoyo y medidas reales de los Gobiernos y los organismos económicos internacionales para contrarrestar y mitigar los efectos del cambio climático de manera sostenible, poniendo los derechos, la dignidad y la calidad de vida de las personas, del presente y del futuro, en el centro.

\section{Referencias bibliográficas}

Arendt, Hannah. Entre el pasado y el futuro. Ocho ejercicios sobre la reflexión política. Barcelona: Ediciones Península, 2016. D0l: https:// doi.org/10.7208/chicago/9780226231785.001.0001.

Arendt, Hannah. Lectures on Kant's Political Philosophy. Chicago: Chicago University Press, 1982.

Benhabib, Seyla. Los derechos de los otros. Extranjeros, residentes y ciudadanos. Barcelona: Editorial Gedisa, 2015.

Camps, Victoria. Tiempo de cuidados. Otra forma de estar en el mundo. Barcelona: Arpa Editores, 2021.

Garcés, Marina. 2022. «Imaginación crítica». Artnodes, no. 29, «Ecologías de la imaginación» (2022).

Gómez, Irene. Deudas pendientes. La justicia entre generaciones. Madrid: Plaza y Valdés - CSIC, 2020.

Kant, Immanuel. Crítica de la razón pura. Madrid: Ediciones Alfaguara, 1996. Kant, Immanuel. Crítica del discernimiento. Madrid: Mínimo Tránsito, 2003.

Marrama0, Giacomo. «Yo múltiple y responsabilidad colectiva.» Vivir para pensar. Ensayos en homenaje a Manuel Cruz (2012), Fina Birulés, Antonio Gómez Ramos y Concha Roldán (eds.). Barcelona: Herder. DOI: https://doi.org/10.2307/j.ctvt9jzgv.26.

Muñoz Sánchez, María Teresa. 2017. «Validez ejemplar y comunidades de certezas. Kant, Arendt, Wittgenstein sobre el poder de juzgar críticamente». Isegoría, no. 57 (2017): 505-31. D0l: https://doi. org/10.3989/isegoria.2017.057.05.

Nussbaum, Martha C. Sin fines de lucro. Por qué la democracia necesita de las humanidades. Buenos Aires/Madrid: Katz Editores, 2010. DOl: https://doi.org/10.2307/j.ctvndv60c.

Nussbaum, Martha C. Paisajes del pensamiento: la inteligencia de las emociones. Barcelona: Paidós, 2008.
Sen, Amartya. «Rational Fools: A Critique of the Behavioral Foundations of Economic Theory». Philosophy and Public Affairs vol. 6, no. 4 (1977): 317-344.

Smith, Adam. La teoría de los sentimientos morales. Madrid: Alianza Editorial, 2009.

Wagner, Astrid. «Libertad estética y libertad práctica: la 'Crítica del discernimiento' y su incidencia en el concepto kantiano de 'libertad moral'». Isegoría, no. 30 (2004): 161-176. Dol: https://doi. org/10.3989/isegoria.2004.i30.481.

Wences Simón, Isabel (ed.). Teoría social y política de la Ilustración escocesa. Madrid/México: Plaza y Valdés, CSIC, 2007.

Zerilli, Linda. El Feminismo y el abismo de la libertad. Buenos Aires: Fondo de Cultura Económica, 2008.

Zerilli, Linda. «The Practice of Judgment: Hannah Arendt's Copernican Revolution.» En: Theory after Theory, Jane Elliott y Derek Atridge (eds.). Londres: Routledge, 2011. 


\section{artnodes}

\section{CV}

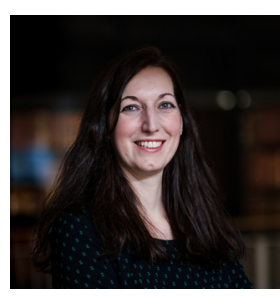

\section{Irene Gómez Franco}

Departamento de Filosofía. Filosofía Moral

Universidad Autónoma de Barcelona

Irene.Gomez.Franco@uab.cat

Filósofa y escritora especializada en filosofía política y moral. Después de cursar sus dos licenciaturas en Barcelona, en Filosofía y en Economía, se doctoró por la Universidad Técnica de Berlín y la Universidad Nacional de Educación a Distancia. Durante su trayectoria académica ha realizado numerosas estancias de investigación, de las que cabe destacar las llevadas a cabo como becaria Marie Curie en la Universidad de Chicago, en tanto que investigadora científica en la Universidad de Potsdam (Alemania) y como profesora invitada en la Universidad Nacional Autónoma de México. Sus publicaciones muestran un compromiso por entender la filosofía como un ejercicio crítico volcado en la práctica, desarrollando su línea de trabajo en torno a dos ejes principales: la teoría de la justicia global (desde un punto de vista espacial y temporal) y el pensamiento feminista. Actualmente, es profesora en la Universidad Autónoma de Barcelona y en la Universitat Oberta de Catalunya, así como investigadora contratada en el Instituto de Filosofía del Consejo Superior de Investigaciones Científicas (Madrid).

https://orcid.org/0000-0002-4086-8184 